\title{
Western North Pacific Typhoons with Concentric Eyewalls
}

\author{
HuNG-CHI KuO \\ Department of Atmospheric Sciences, National Taiwan University, Taipei, Taiwan \\ CHIH-Pei CHANG \\ Department of Meteorology, Naval Postgraduate School, Monterey, California \\ YI-TING YANG AND HAU-JANG JIANG \\ Department of Atmospheric Sciences, National Taiwan University, Taipei, Taiwan
}

(Manuscript received 4 November 2008, in final form 19 April 2009)

\begin{abstract}
This study examines the intensity change and moat dynamics of typhoons with concentric eyewalls using passive microwave data and best-track data in the western North Pacific between 1997 and 2006. Of the 225 typhoons examined, 55 typhoons and 62 cases with concentric eyewalls have been identified. The data indicate that approximately $57 \%$ of category 4 and $72 \%$ of category 5 typhoons possessed concentric eyewalls at some point during their lifetime. While major typhoons are most likely to form concentric eyewalls, the formation of the concentric structure may not be necessarily at the lifetime maximum intensity. Approximately onethird of concentric eyewall cases are formed at the time of maximum intensity.

The moat is known to be heavily influenced by the subsidence forced by the two eyewalls. Rozoff et al. proposed that the rapid filamentation dynamics may also contribute to the organization of the moat. This paper examines the possibility of rapid filamentation dynamics by devising a filamentation moat width parameter. This parameter can be computed from the best-track typhoon intensity and the passive microwave satellite-estimated inner eyewall radius for each typhoon with concentric eyewalls. The filamentation moat width explains $40 \%$ of the variance of the satellite-observed moat width in the group with concentric eyewall formation intensity greater than $130 \mathrm{kt}$.

The typhoon intensity time series in both the concentric and nonconcentric composites are studied. The time series of intensity is classified according to the 24-h intensity change before and after the concentric eyewalls formation. The averaged concentric eyewall formation latitudes in the groups with negative intensity change before concentric eyewall formation are at higher latitudes than that of the positive intensity change groups. Intensity of the concentric typhoons tends to peak at the time of secondary eyewall formation, but the standard model of intensification followed by weakening is valid for only half of the cases. Approximately $74 \%$ of the cases intensify $24 \mathrm{~h}$ before secondary eyewall formation and approximately $72 \%$ of the cases weaken $24 \mathrm{~h}$ after formation. The concentric composites have a much slower intensification rate $12 \mathrm{~h}$ before the peak intensity (time of concentric formation) than that of the nonconcentric composites. For categories 4 and 5, the peak intensity of the concentric typhoons is comparable to that of the nonconcentric typhoons. However, $60 \mathrm{~h}$ before reaching the peak the concentric composites are $25 \%$ more intense than the nonconcentric composites. So a key feature of concentric eyewall formation appears to be the maintenance of a relatively high intensity for a longer duration, rather than a rapid intensification process that can reach a higher intensity.
\end{abstract}

Corresponding author address: Prof. Hung-Chi Kuo, Department of Atmospheric Sciences, National Taiwan University. No. 1, Sec. 4, Roosevelt Rd., Taipei 10617, Taiwan.

E-mail: kuo@as.ntu.edu.tw

DOI: $10.1175 / 2009 M W R 2850.1$

(c) 2009 American Meteorological Society 


\section{Introduction}

Tropical cyclones (TCs), and particularly strong TCs are observed with a concentric eyewall (CE) structure that has an inner eyewall and an outer eyewall separated by a convective minimum region. The secondary eyewalls ${ }^{1}$ are generally identified as quasi-circular rings of convective cloud. The outer eyewalls are often, but not always, collocated with the secondary wind maximum (Samsury and Zipser 1995), analogous to the collocation of the peak winds and the primary eyewall convection. Eyewall replacement cycles in such TCs are very important for accurate forecasts of rapid intensity change (Willoughby 1990; Black and Willoughby 1992). Maclay et al. (2008) identify secondary eyewall formation and eyewall replacement cycles as an internally dominant process that is responsible for structure change and thus the low-level area-integrated kinetic energy. In the past decade, the availability of satellite passive microwave data has made it possible to reveal the CE structure in TCs that had previously been difficult with only visible and infrared images. From a study of passive microwave data taken between 1997 and 2002, Hawkins and Helveston (2004) concluded that CE structure exist in a much higher percentage of TCs than had previously been estimated using visible and infrared satellite sensors. Although based on a relatively small sample, their results suggest that approximately $40 \%$ of the Atlantic, $60 \%$ of the eastern Pacific, and $80 \%$ of the western Pacific intense storms [maximum wind $>120 \mathrm{kt}$ $\left.\left(\sim 62 \mathrm{~m} \mathrm{~s}^{-1}\right)\right]$ have CE structures. The preponderance of large-radius $\mathrm{CE}$ in the western Pacific compared to other basins was further suggested by Hawkins et al. (2006). Kossin and Sitkowski (2009) designed a new empirical model that provides forecasters a probability of imminent $\mathrm{CE}$ formation. They identified the CE structure through a subjective visual analysis of passive microwave satellite images from the Naval Research Laboratory in Monterey covering the period of 1997-2006. In their paper, CE climatology for the North Atlantic and the central and eastern North Pacific are given. Their results of CE percentage of major hurricanes (categories 3-5) in the Atlantic $(70 \%)$ and eastern Pacific (35\%) are in general agreement with that of Hawkins et al. (2006).

Both the internal and external dynamics may play important roles in CE formations. Examples of internal dynamics are propagating vortex Rossby waves that interact with critical radius (Montgomery and Kallenbach 1997) and axisymmetrization of binary vortex interaction (Kuo et al. 2004, 2008). These dynamics highlight

\footnotetext{
${ }^{1}$ We use the "outer eyewall" and "secondary eyewall" interchangeably in the paper.
}

the importance of the strong core vortex. In particular, Kuo et al. (2008) discussed the role vorticity skirts outside the TC core may play in the formation of CE structure of various sizes through the binary vortex interaction mechanism. On the other hand, Nong and Emanuel (2003) showed that the CE may form due to some favorable ambient environment conditions or external forcing and wind-induced surface heat exchange (WISHE) instability.

A key feature of the CEs is the formation of the moat, which is the space between the inner and outer eyewalls where subsidence is observed (e.g., Dodge et al. 1999). Houze et al. (2007) presented aircraft observations that illustrated how the temperature and moisture profiles in the moat can evolve into a vertical structure closely resembling those found in the eye. It is generally recognized that the moat formation mechanism is heavily influenced by the subsidence forced by the two eyewalls (Dodge et al. 1999). On the other hand, Rozoff et al. (2006) proposed the idea of a rapid filamentation zone outside the radius of maximum wind of TCs. The rapid filamentation zone is a region of strain-dominated flow where the filamentation time is smaller than the $30-\mathrm{min}$ moist convective overturning time. Deep convection in the rapid filamentation zone may become highly distorted and even suppressed, leading to the moat formation. Through a numerical modeling study Wang (2008) found that the rapid filamentation process can effectively suppress high-azimuthal wavenumber asymmetries immediately outside the radius of maximum wind but has little effect on well-organized low wavenumber spiral bands. He argued that the rapid filamentation may play a secondary role in the formation of the moat in TCs. However, when a TC becomes very strong it is possible that the subsidence is more likely confined to the edge of the deep convection and strengthened by inertial stability (e.g., Rozoff et al. 2008). With a moat region of sufficiently large size, the impact of subsidence is unlikely to be uniform throughout the moat, which leaves the possibility that the rapid filamentation dynamics may contribute to the organization of the moat. This is more likely to happen in the western North Pacific because of the presence of very intense typhoons and large-radius CE cases (Hawkins et al. 2006; Kuo et al. 2008).

This study, using passive satellite microwave and besttrack datasets, examines the intensity change and the moat rapid filamentation dynamics of western North Pacific CE typhoons between 1997 and 2006. Section 2 describes the data, the analysis method, and the climatology. The results regarding the relationship between moat size and filamentation dynamics are discussed in section 3 , and those related to the intensity change issues are discussed in section 4 . Section 5 is a summary. 


\section{Data, method, and climatology}

We used the best-track dataset from the Joint Typhoon Warning Center (JTWC) and microwave image sequences to examine the characteristics of typhoons with CE in the western North Pacific in the period of 1997 2006. The microwave data is the passive SSM/I $85-\mathrm{GHz}$ horizontal polarized orbital images obtained from the Naval Research Laboratory (NRL) Marine Meteorology Division in Monterey, California (Hawkins et al. 2001). The NRL Web site also provides the passive Tropical Rainfall Measuring Mission (TRMM) Microwave Imager (TMI) data from the polar-orbiting TRMM satellite (Kummerow et al.1998). The coverage of the TMI data in general is smaller than the SSM/I data but the TMI data resolution is higher than the SSM/I data. The best-track data from JTWC uses the Geostationary Meteorological Satellite (GMS) infrared and visible cloud images as the data source for the Dvorak technique to estimate intensity. The maximum 1-min sustained wind speed was derived using the Dvorak T number.

There appears to be no uniform and objective definition of $\mathrm{CE}$ event from microwave satellite. A quasi-circular outer ring of convection that is clearly separated from the primary eyewall has been the common way to define CE case. We adopted a criterion that is based on the outer eyewall deep convection (with a total blackbody temperature $\mathrm{Tbb}$ approximately $230 \mathrm{~K}$ ) covering at least two-thirds of a circle. This is stricter than some previous studies using one-half of a circle as the criterion. The same criterion is applied to satellite data with partial coverage of the passive microwave satellite data. Thus, it is possible that we missed some CE typhoons because of the partial microwave satellite coverage. The critical issues in the CE identification are the values of Tbb in the deep convection and the symmetry of the outer eyewalls. If we choose a Tbb of higher value around 235-240 K, we could easily double our CE cases. On the other hand, if we choose a more strict Tbb around $225 \mathrm{~K}$, we will have just half as many of the cases with Tbb $230 \mathrm{~K}$. Our method is in general similar to Hawkins et al. (2001) in defining the CE cases except they used one-half circle as the minimum. Our choices are more on the conservative side while retaining enough cases for the statistics. There were 243 typhoons in the western North Pacific during 1997-2006, of which 18 typhoons were removed for missing microwave coverage for more than $24 \mathrm{~h}$. A total of 55 typhoons and 62 cases $(7$ typhoons with double CE cases) were identified to possess CE structure (analyzed data of CE cases are available online at http:// faculty.nps.edu/cpchang/papers/CEdataWPAC9706.pdf). Figure 1 gives examples of marginally accepted and rejected cases.
The microwave data are used to define the CE structure, the inner eyewall radius, moat width, and the $\mathrm{CE}$ formation time. The moat is the high brightness temperature region between the eyewalls. The eyewall radius and the moat width are determined by averaging the measured values from eight radial arms. Limited experiments with a 16-radial-arm average were found to yield similar results. The best-track data provide the typhoon intensity, intensity change, location, and lifetime maximum intensity. The JTWC best track is in 6-h time resolution. We account for the irregular satellite observation times with the regular JTWC best-track data by matching the time closest to the satellite observation. These data are used to compute the CE formation percentage and analyze the relationships between moat and intensity, CE, and intensity change, and compare the intensity changes of concentric typhoons with the nonconcentric typhoons. Note that the two datasets used are not totally independent as JTWC considered the microwave imageries (such as SSM/I and TMI) in producing the best-track data. However, using them together remains the only way to deduce a quantitative estimate of the formation percentages and their distribution relative to intensity.

Figure 2 gives the locations of $\mathrm{CE}$ formation events in the western North Pacific between 1997 and 2006. Figure 2 suggests that strong typhoons containing CE structure tend to occur farther west in the basin. This may be because typhoons tend to be stronger in the western part of the basin after a long journey over ocean. Figure 3 gives the number and the average latitude of $\mathrm{CE}$ formation by month. The majority of typhoons with CE structure ( 54 cases out of the total 62 cases) form between June and October. Most of the CE cases (41 out of 62) are found with formation intensity at categories 4 and 5. Figures 2 and 3 indicate a northern seasonal migration of CE structure formation locations. Between June and October, the weaker intensity cases tend to locate at higher latitudes than the stronger intensity cases. It is possible that external forcings (e.g., Nong and Emanuel 2003) may be important for the higher-latitude $\mathrm{CE}$ with weaker formation intensity, but the specifics remain to be studied.

Figure 4a shows a histogram of the CE cases for each typhoon intensity category according to the SaffirSimpson scale. Of the 225 typhoons examined, approximately one-quarter (55 typhoons) possessed the CE structure in which $83 \%$ or 47 typhoons reached categories 4 and 5 . Specifically, $57 \%$ of category 4 and $72 \%$ of category 5 typhoons possessed CE structure at some point during their lifetime. This result agrees with the general notion that $\mathrm{CE}$ structures are associated with TCs of sufficient strength. The results are also in general agreement with the North Atlantic climatology of Kossin and Sitkowski (2009), who found that one-third of all 
Yagi 2006

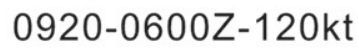

0920-0729Z

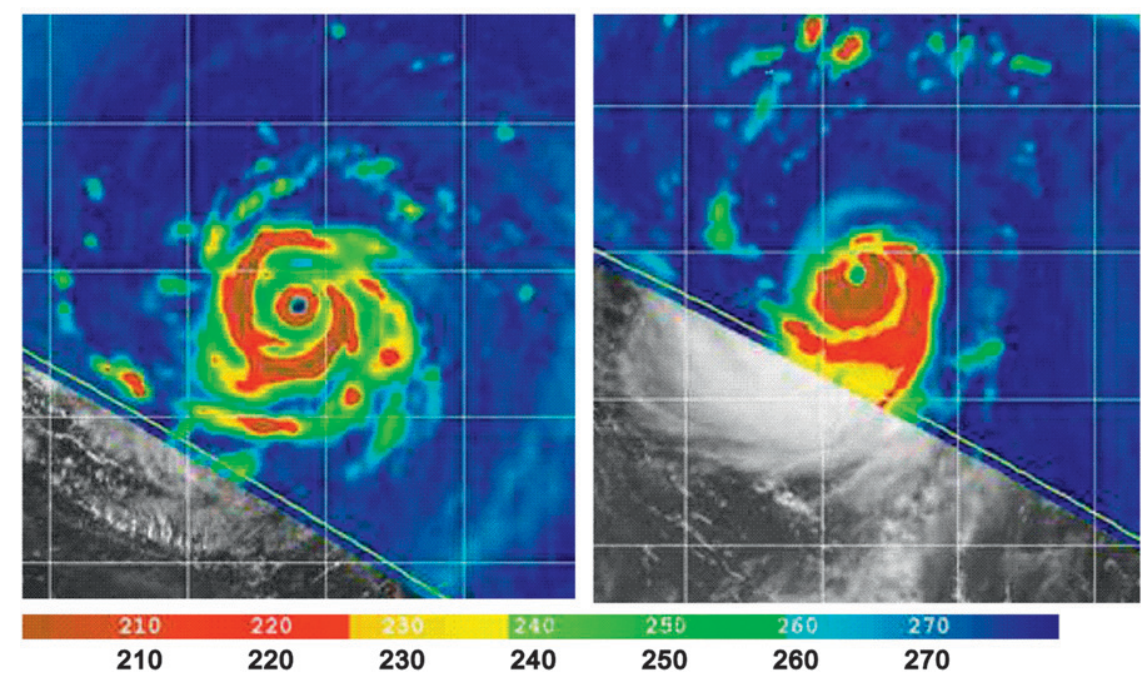

FIG. 1. Color-enhanced microwave images of Typhoon Yagi and Typhoon Saomai in 2006. Typhoon Yagi is identified as concentric case while Typhoon Saomai is classified as nonconcentric case. Saomai's maximum intensity was $140 \mathrm{kt}$ at 0809-1200 UTC. hurricanes developed a CE structure at least once during their lifetime. Comparatively, $70 \%$ of major hurricanes (categories 3-5) were observed to form CE structures at least once in their lifetime. They also reported that a hurricane is apparently more likely to form a $\mathrm{CE}$ structure in July or September than other months.

Figure $4 \mathrm{~b}$ is similar to Fig. 4a except the horizontal coordinate is the intensity at the time of secondary eyewall formation rather than the maximum intensity of each typhoon. Secondary eyewalls are observed to form within a broad range of intensities. Because of the inclusion of the 7 typhoons with 2 concentric formation cases, the total number of CE cases in Fig. $4 \mathrm{~b}$ is 62 rather than the 55 in Fig. 4a. Figure $4 b$ indicates that $66 \%$ of the $\mathrm{CE}$ cases formed at categories 4 and 5 strengths; the percentage is lower than what is indicated in Fig. 4a. This implies that while the CE structure often forms in typhoons of sufficient strength, they may not necessarily form at the peak intensity of a typhoon. An analysis of the time of $\mathrm{CE}$ formation relative to the time of lifetime maximum intensity is given in section 4 .

\section{Moat size and filamentation dynamics}

Figure 5 shows four scatter diagrams involving the moat width $\left(d_{0}\right)$, the cyclone core size in terms of inner eye radius $\left(r_{0}\right)$, and the cyclone intensity at CE structure formation $\left(V_{m}\right)$. Figure 5a is moat width versus core size,
Fig. $5 \mathrm{~b}$ is core size versus cyclone intensity, Fig. $5 \mathrm{c}$ is moat width versus estimated cyclone core vorticity strength ( $\zeta_{0}=2 V_{m} / r_{0}$, with the solid-body rotation assumption) and Fig. $5 \mathrm{~d}$ is moat width versus cyclone intensity. The green, black, and red circles indicate categories 3, 4, and 5, respectively. The filled circles are the averaged values for the respective categories. Note that the analysis indicates that the mean satellite-estimated moat width $d_{0}$ is $33.5 \mathrm{~km}$ and the standard deviation is $21.7 \mathrm{~km}$. In general, the large scatters in these diagrams indicate no significant relationships among the moat width, core size, core vorticity, and intensity for the CE typhoon cases. This lack of relationship among the kinematic parameters seems to suggest that dynamic processes are not important for moat formation. We will show in this section that the rapid filamentation dynamics may still contribute to the organization of the moat in very strong typhoons.

Observational studies (e.g., Dodge et al. 1999; Houze et al. 2007) have demonstrated the important roles of subsidence in moat formation physics. Rozoff et al. (2006) proposed that a rapid filamentation zone outside the radius of maximum wind in strong tropical cyclones may also play a role. The rapid filamentation zone is a region of strain-dominated flow where the filamentation time is shorter than the typical moist convective overturning time. Without adequate time for organization, deep convection in the rapid filamentation zone may 


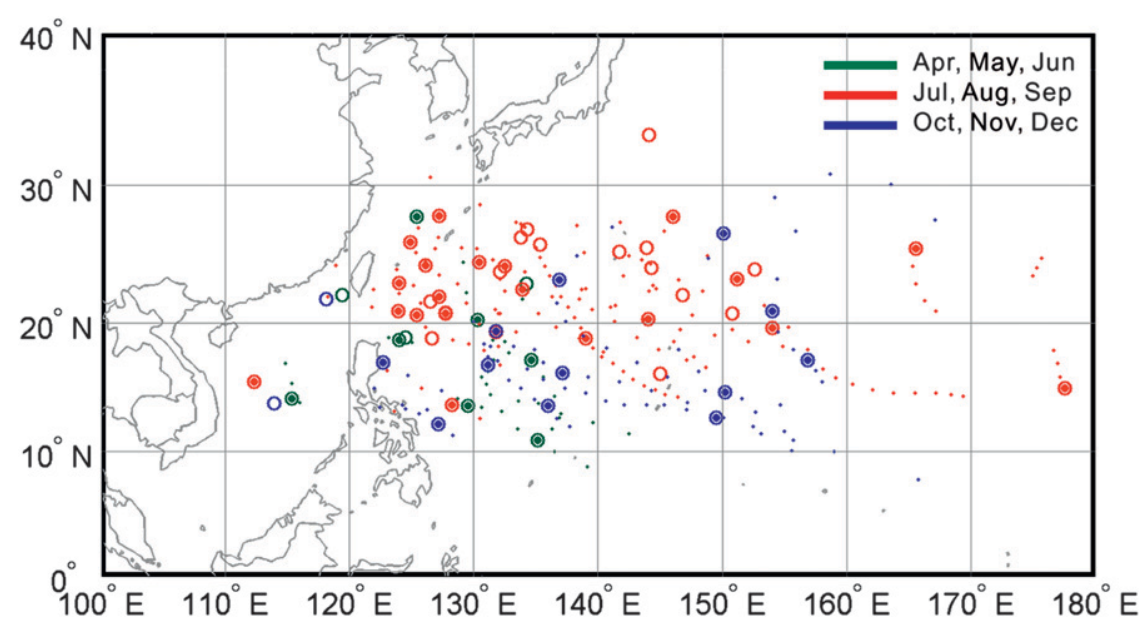

FIG. 2. Locations of concentric eyewall formation events in the western North Pacific between 1997 and 2006. The seasonal cycle of concentric eyewall cases is represented by green (April, May, and June), red (July, August, and September), and blue (October, November, and December) colors. The dots display the locations of typhoons with intensity greater than or equal to category 4 on the Saffir-Simpson scale. The circles indicate the locations of concentric eyewall formation. A dot inside a circle indicates concentric eyewall formation with intensity greater than or equal to category 4 .

become highly distorted and even suppressed, leading to the formation of a moat. The strain effect associated with the vortex will likely decrease the probability of secondary eyewall formation within a certain radial distance of the primary eyewall, thus, it may become a factor in determining the moat width.

To evaluate the role that rapid filamentation dynamics may play in the moat formation mechanism, we devise a scheme to compare the moat width predicted by the rapid filamentation dynamics to that observed from the microwave satellite data. Mallen et al.(2005) aircraft flightlevel data analysis shows that TCs are often characterized by a relatively slow decrease of tangential wind outside the radius of maximum wind, or a corresponding cyclonic vorticity skirt. We assume that the vortex is in solid-body rotation inside the radius of maximum wind and the tangential wind decreases according to $r^{-\alpha}$ outside of the radius of maximum wind, where $\alpha$ is the skirt parameter. Aircraft observations of the azimuthal winds in TCs (e.g., Shea and Gray 1973; Mallen et al. 2005) suggest that a reasonable range for the skirt parameter is $0.5 \leq \alpha \leq 1$.

Starting with the best-track intensity $V_{m}$ and satelliteestimated core size $r_{0}$, the core vorticity strength is $\zeta_{0}=$ $2 V_{m} / r_{0}$, and the total strain rate in terms of the vorticity is $S_{1}^{2}+S_{2}^{2}=[(1+\alpha) / 2]^{2} \zeta_{0}^{2}\left(r_{0} / r\right)^{2+2 \alpha}$. The filamentation time based on Rozoff et al. (2006) is $\tau_{\text {fil }}=2\left(S_{1}^{2}+S_{2}^{2}-\right.$ $\left.\zeta^{2}\right)^{-1 / 2}$ for $S_{1}^{2}+S_{2}^{2}>\zeta^{2}$, where $\zeta$ is the vorticity at the moat. Thus, $S_{1}^{2}+S_{2}^{2}-\zeta^{2}=\alpha \zeta_{0}^{2}\left(r_{0} / r\right)^{2+2 \alpha}$, and the filamentation time is $\tau_{\text {fil }}=2\left(r / r_{0}\right)^{1+\alpha} /\left(\sqrt{\alpha} \zeta_{0}\right)$. We then define the convective overturning time $\tau_{\text {con }}=30 \mathrm{~min}$, which is the time for a parcel traveling from the top of the boundary layer $(600 \mathrm{~m})$ to cloud top $(15 \mathrm{~km})$ with a typical speed $8 \mathrm{~m} \mathrm{~s}^{-1}$, or for a weaker updraft of $3-4 \mathrm{~m} \mathrm{~s}^{-1}$ that extends slightly above freezing level before merging into a

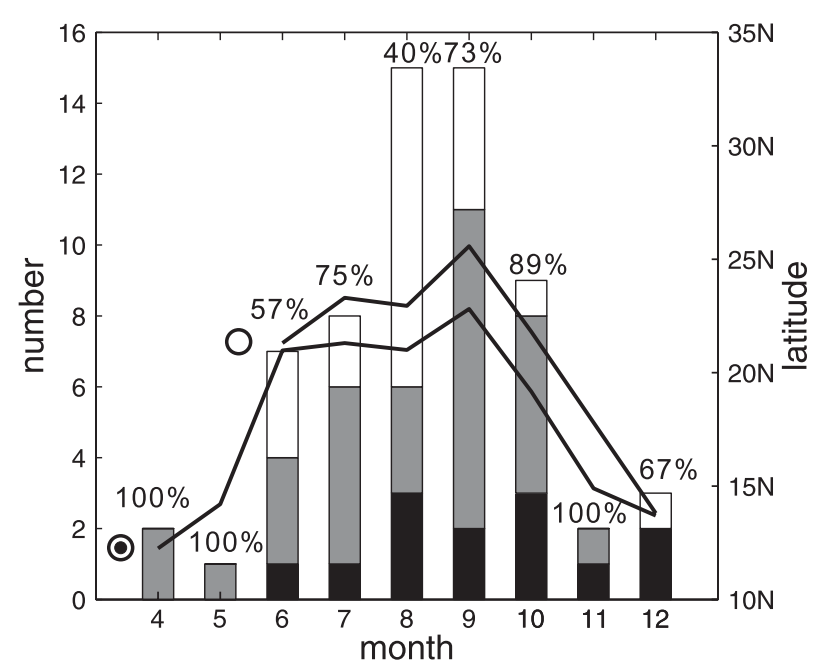

FIG. 3. The number and the average latitude of concentric eyewall formation by month. The black, gray, and white bars represent the number of concentric eyewall cases of category 5 , category 4 , and less than category 4 intensity. The upper (lower) curve with circle (a dot inside the circle) indicates the average latitude of concentric eyewall formation with intensity less than Category 4 (greater than or equal to category 4) by month. The percentages by month on top of the diagrams are the percentage of concentric eyewall typhoons with intensity greater than or equal to category 4. 

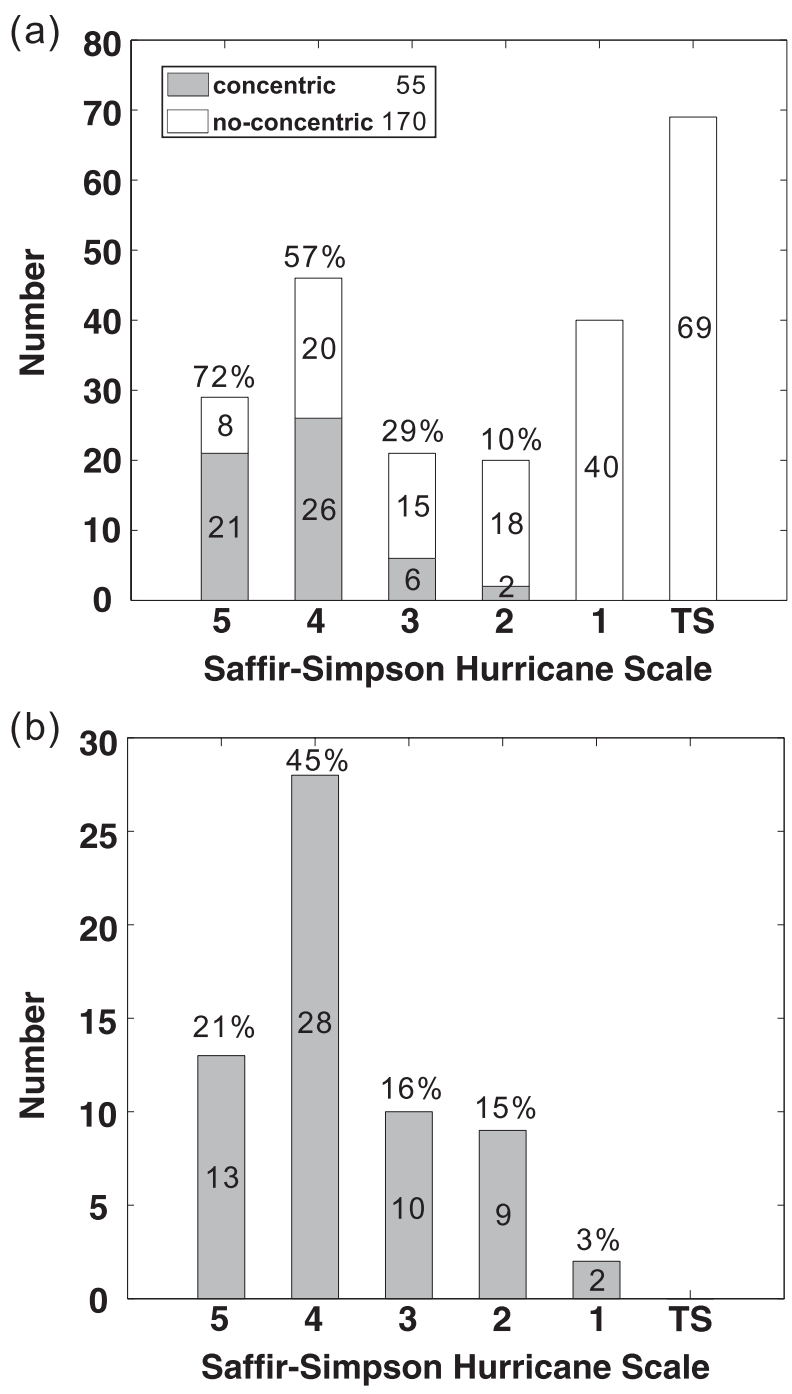

FIG. 4. (a) Histogram of the concentric cases against the typhoon intensity as categorized by the Saffir-Simpson scale. (b) As in Fig. 3a, but against the typhoon intensity at the time of secondary eyewall formation.

region of general mesoscale lift. We then define the rapid filamentation radius $r^{*}$ as a radius where the filamentation time is set to equal to the convective overturning time $\tau_{\text {con }}=2\left(r^{*} / r_{0}\right)^{1+\alpha} /\left(\sqrt{\alpha} \zeta_{0}\right)$, and $r^{*}=\left(\sqrt{\alpha} \zeta_{0} \tau_{\text {con }} / 2\right)^{1 /(1+\alpha)} r_{0}$. Because the filamentation time is smaller (larger) than the convective overturning time inside (outside) the radius $r^{*}$, the width of the rapid filamentation zone is $d_{\mathrm{ft}}=r^{*}-r_{0}=\left[\left(\sqrt{\alpha} \zeta_{0} \tau_{\mathrm{con}} / 2\right)^{1 /(1+\alpha)}-1\right] r_{0}$.

In summary, the rapid filamentation zone is a function of the convective overturning time, the vorticity determined from the best-track intensity, the satellite estimated core size, and the skirt parameter.

Figure 6 gives the variance $\left(R^{2}\right)$ of the observed nondimensional moat width that is explained by the nondimensional filamentation zone width. The nondimensional width calculation is according to the core size $r_{0}$ in each CE typhoon. Figure 6a gives the moat width variances explained by the nondimensional filamentation moat width versus cyclone intensity. We calculated the $R^{2}$ for concentric cases with formation intensities greater than, and less than and equal to, a set intensity that ranges from 85 to $135 \mathrm{kt}$ (in 5-kt intervals as plotted on the abscissa). The constant skirt parameters used in the calculations are $1,0.5$, and 0.3 . The 0.3 value corresponds to a very slow outward decay of tangential wind from the core. Figure 6a suggests that, regardless the skirt values used, the filamentation moat width explains about $40 \%$ of the variance of the satellite-observed moat width in the group with $\mathrm{CE}$ formation intensity greater than $130 \mathrm{kt}$ (19 cases). We have also performed tests by randomly assigning different skirt parameter values between 0.3 and 1 to each of these 19 cases and the results still yield the similar $40 \%$ variance that is explained by the filamentation moat width. The scatterplot and the variance explained for CE formation intensity of categories 5,4 , and $3(\alpha=0.5)$ are shown in Figs. 6b,c,d, respectively. The $R^{2}$ value in category 3 (Fig. 6d) is insignificant. The $R^{2}$ value in category 4 (Fig. 6c) is 0.13 as it covers the TC formation intensity from 114 to $135 \mathrm{kt}$. The $R^{2}$ value in Fig. $6 \mathrm{a}$ is approximately 0.2 for all the TCs stronger than $115 \mathrm{kt}$.

Figure 6 suggests that the filamentation dynamics appears to be relevant in the group with the strongest CE formation intensity. Rozoff et al. (2008) showed that the subsidence in the moat may become most important in the later stages of $\mathrm{CE}$ formation. The strengthening convection in the outer eyewall may lead to enhanced up-down circulation and inertial stability, which in turns leads to the narrowing (or confinement) of up-down circulation that enhances the local subsidence. When the strong subsidence is confined by inertial stability to the edge of the deep convection for a very strong TC, the impact of subsidence will be less likely uniform in the moat region if the moat is larger than the narrow subsidence zone. This leaves the possibility for the rapid filamentation in strong CE typhoons to affect the organization of the moat in the early stage. This is more likely to happen in the western North Pacific because of the prevalence of large-radius CE cases (Hawkins et al. 2006; Kuo et al. 2008).

\section{Intensity change}

The evolution of intensity around CE formation is analyzed by compositing with respect to the CE formation time. The time series of intensity is classified into four groups: PN, PP, NP, and NN, according to the 24-h intensity change before and after the formation. Here the 

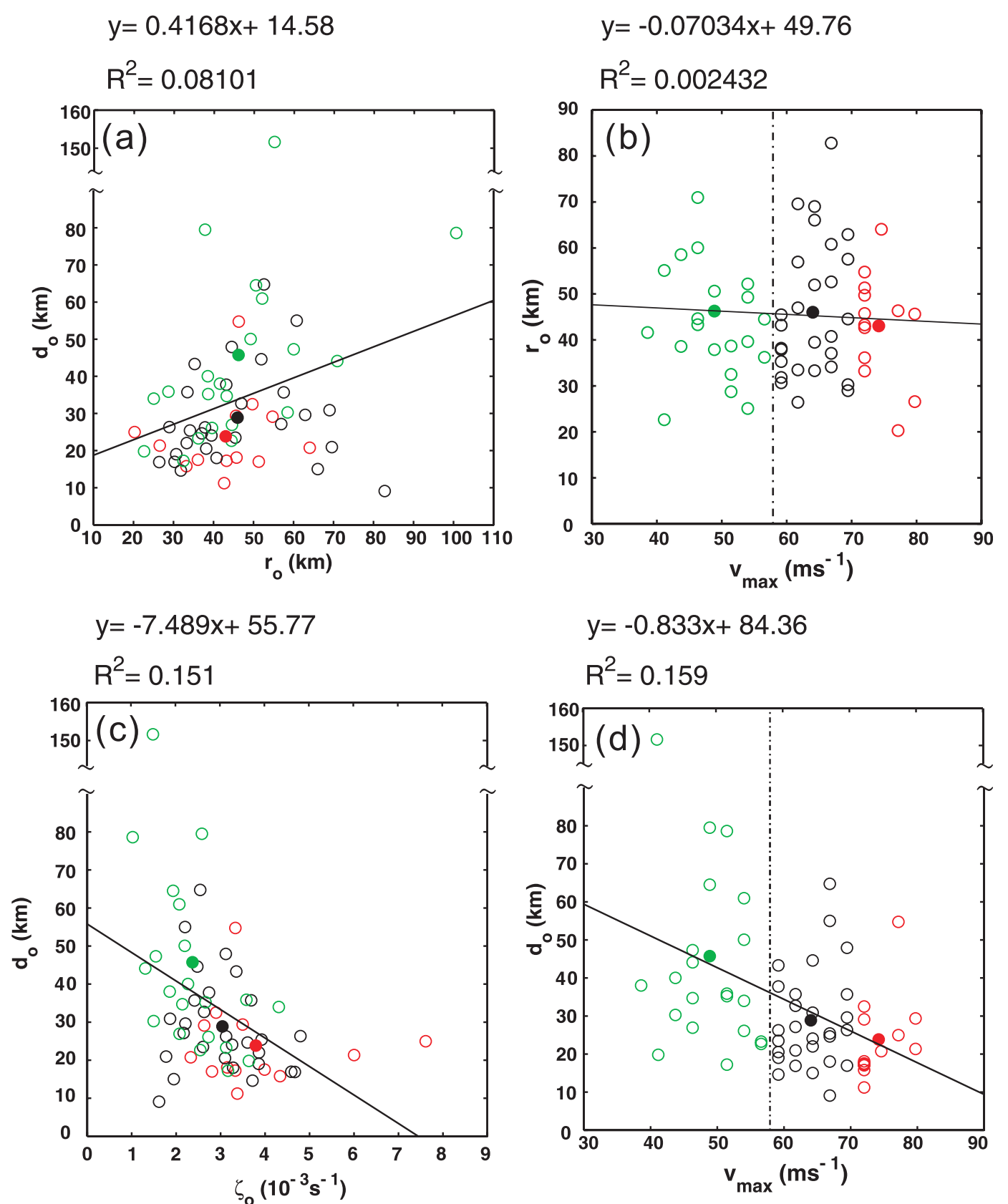

FIG. 5. Scatter diagram of (a) moat width vs cyclone core size, (b) cyclone core size vs cyclone intensity, (c) moat width vs estimated cyclone core vorticity strength, and (d) moat width vs cyclone intensity for the concentric eyewall cases studied. The green, black, and red circles indicate categories 3, 4, and 5, respectively. The cyclone core size and the moat width are determined from microwave data and the intensity from the best-track data. The cyclone core vorticity is estimated from the core size and the intensity. Note the wide scatter of values.

letter $\mathrm{N}$ indicates negative intensity trend and the letter $\mathrm{P}$ indicates positive intensity trend. The first letter in each group's name indicates the intensity change in the last $24 \mathrm{~h}$ prior to formation and the second letter the intensity change during the first $24 \mathrm{~h}$ after the formation. For example, the PN group showed strengthening prior to formation and weakening after the CE formation, all within $24 \mathrm{~h}$ of the formation time. The above groups do not include cases in which the typhoon center is within $200 \mathrm{~km}$ from land, so that weakening due to land effects is excluded.

While strong typhoons are more likely to form a CE structure, Fig. 4 indicates that the CE structure may not necessarily form when the typhoons are at their peak intensity. Figure 7 is an analysis of the CE formation 
(a)

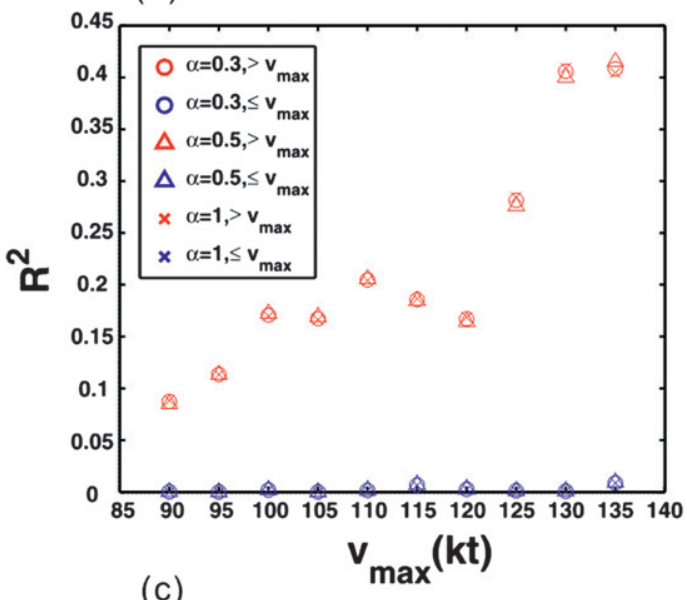

(c)

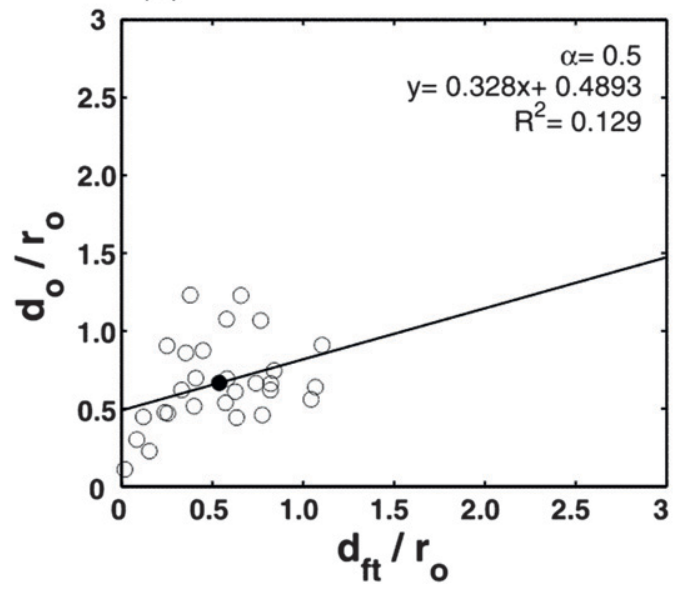

(b)

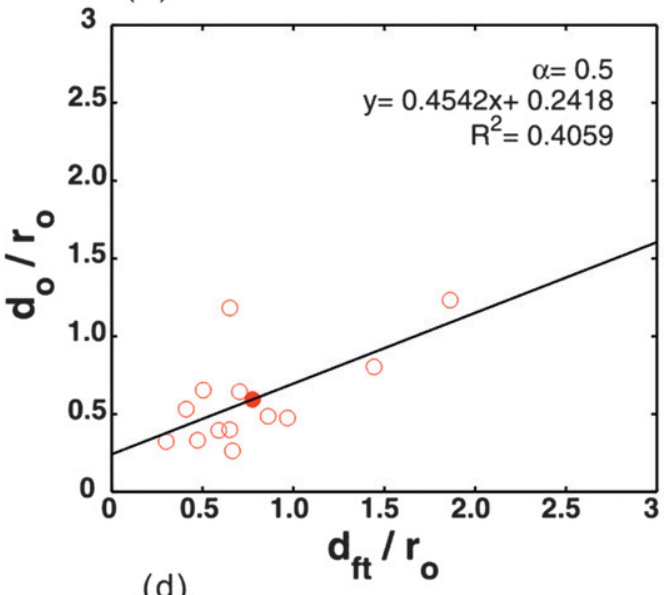

(d)

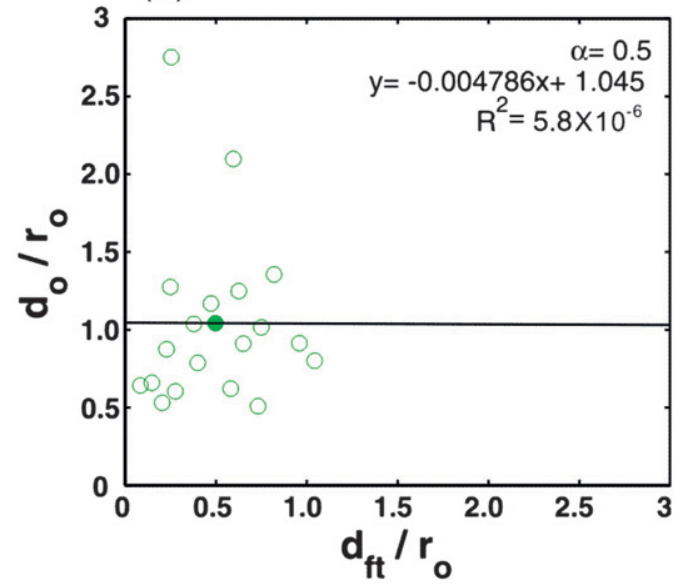

FIG. 6. (a) Scatter diagram of $R^{2}$, which is the variance of the nondimensional moat width explained by the nondimensional filamentation moat width vs cyclone intensity. The red and blue colors indicate, respectively, $R^{2}$ for concentric cases with intensities greater than, and less than and equal to, a set intensity given on the abscissa. The circles, triangles, and crosses display $\alpha=0.3, \alpha=0.5$, and $\alpha=1$, respectively. (b) Scatter diagram of nondimensional moat width vs of the nondimensional filamentation moat width for concentric cases in category 5 . The vortex structure is assumed with a skirt parameter of 0.5. (c) As in (b), but for concentric cases in category 4. (d) As in (b), but for concentric cases in category 3 .

time relative to the time of lifetime maximum intensity. Approximately one-third of CE cases (20 cases) are formed at the time of maximum intensity and slightly less than one-quarter of the cases ( 15 cases) formed within $30 \mathrm{~h}$ after the typhoon's lifetime maximum intensity. There are 23 (out of 62) cases in which the CE structure forms $30 \mathrm{~h}$ after the maximum intensity time. Of these cases, 6 are multiple CE formation cases; and 11 are formed within $24 \mathrm{~h}$ of the typhoon's second intensity peak. Thus, half of the cases ( 31 cases in the PN group) reach peak intensity at the $\mathrm{CE}$ formation time.

Figure 8 gives the composite time series of intensity as well as the percentage of NP, NN, PN, and PP cases. The standard deviation in these composites is on the order of $15 \mathrm{kt}$. More than half $(51 \%)$ of the CE formation cases are in the $\mathrm{PN}$ group in which the intensity increases
$24 \mathrm{~h}$ before the CE formation and followed by a weakening in the next $24 \mathrm{~h}$. It is noted that 19 of the 24 typhoons in this group are of categories 4 and 5 at $\mathrm{CE}$ formation. The averaged composite and the PN group agree with the typical notion that the CE structure often forms in stronger tropical cyclones and those tropical cyclones often weaken after a secondary eyewall forms.

Besides the PN group, most of the CE cases are in the PP and NN groups with 12 and 11 cases, respectively. The NP group has only two cases. Figure 8 indicates that the intensity increase after the CE formation in the NP and $\mathrm{PP}$ groups is not as significant as that prior the $\mathrm{CE}$ formation. It is more appropriate to read the second $\mathrm{P}$ in our classification as the typhoons maintaining their intensity in the $24 \mathrm{~h}$ after the CE formation. Note that the PP group has a mean $\mathrm{CE}$ formation intensity approximately 
(a)

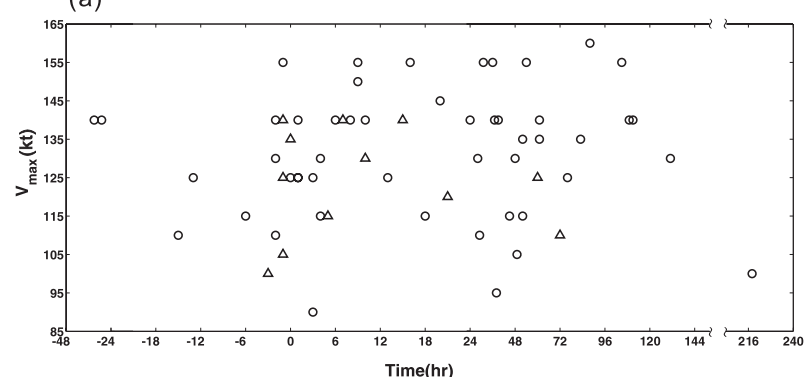

(b)

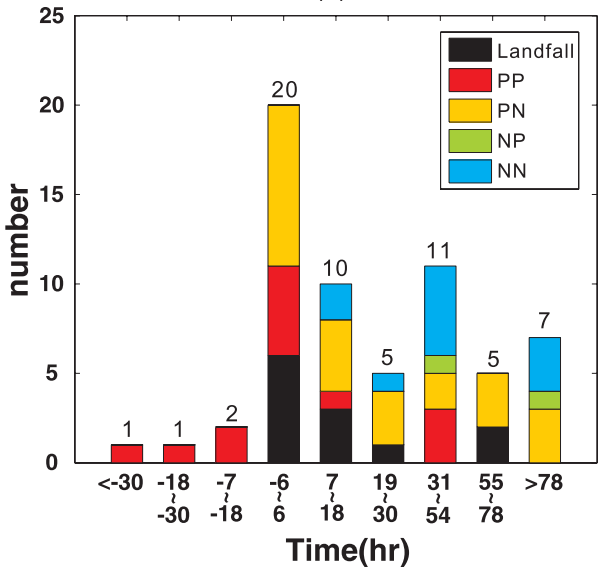

FIG. 7. (a) The concentric forming intensity and the difference between the formation time and the maximum intensity time $(0 \mathrm{~h})$. The triangles indicate cases that prior to or after 24-h concentric eyewall formation, the cyclone center about $200 \mathrm{~km}$ near the land. The circles display the no land influence cases. (b) Histogram of the concentric cases against the difference between the concentric eyewall formation time and the maximum intensity time in PN, PP, $\mathrm{NN}, \mathrm{NP}$, and landfall categories. The negative (positive) values indicate that the formation time is before (after) the maximum intensity time.

$10 \mathrm{~m} \mathrm{~s}^{-1}$ stronger than that in the NP group, with 7 out of 12 cases at categories 4 and 5 intensity at CE formation. On the other hand, the NP group has the weakest CE formation intensity, and 9 out of the 13 cases in the $\mathrm{NN}$ and NP groups have $\mathrm{CE}$ formation intensity of categories 2 and 3. The majority of typhoons in the NN and NP groups do not have peak intensity at their CE formation time. Overall, while only half of the cases peak at the time of $\mathrm{CE}$ formation, approximately $73 \%$ of the cases intensify $24 \mathrm{~h}$ before CE formation and approximately $71 \%$ of the cases weaken $24 \mathrm{~h}$ after $\mathrm{CE}$ formation.

An example of the structure from each of the four groups is given in Fig. 9 by displaying the color-enhanced microwave images before, at, and after $\mathrm{CE}$ formation and the corresponding intensity time series. The time difference between the $\mathrm{CE}$ formation and maximum intensity is also shown. The microwave imagery in the PN case agrees with the typical eyewall replacement cycle model (e.g., Black and Willoughby 1992), in which the

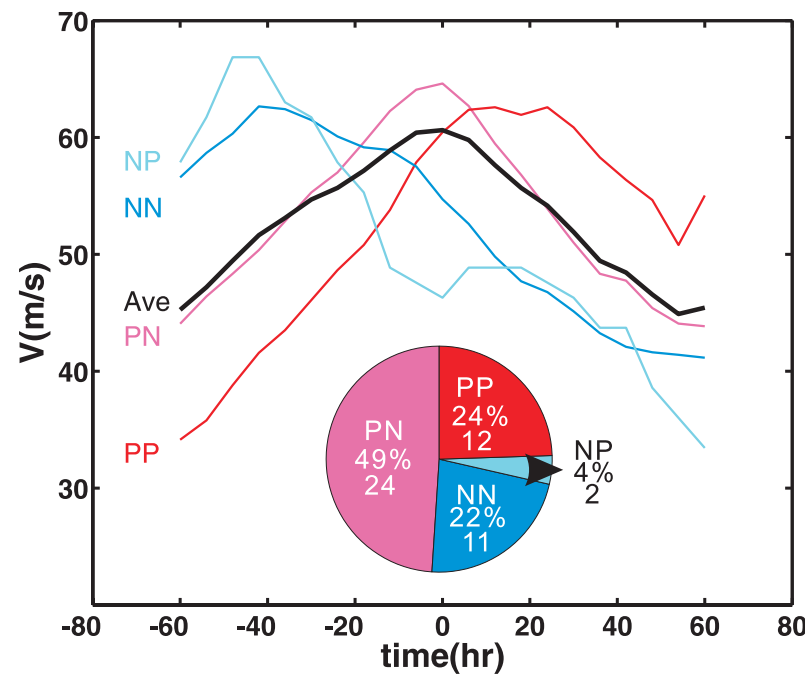

FIG. 8. Composite time series of the intensity for NP, NN, PN, and PP groups. The composite was done with respect to the secondary eyewall formation time $(0 \mathrm{~h})$. The percentage of each case is also shown. The first letter $\mathrm{N}(\mathrm{P})$ represents that the intensity decreases (increases) $24 \mathrm{~h}$ before the concentric eyewall formation. The second letter $\mathrm{N}(\mathrm{P})$ represents that the intensity decreases (increases) $24 \mathrm{~h}$ after the concentric eyewall formation. More than half of the concentric eyewall formation cases are in the PN group, where the intensity increases (decreases) before (after) the concentric eyewall formation.

inner eyewall dissipates after the secondary eyewall forms, and the intensity decreases. However, the PP, NN, and NP cases do not fit this model.

Table 1 shows the averages and standard deviations of vertical wind shear (850-200 and 850-500 hPa) and sea surface temperature (SST) of these groups based on the Statistical Hurricane Intensity Prediction Scheme (SHIPS; DeMaria et al. 2005) data. There are 7 cases with incomplete vertical shear and SST data and the table covers around $85 \%$ of our dataset. We have also included the averaged formation latitudes for the groups. Depending on the intensity change $24 \mathrm{~h}$ before the CE formation, there appears to be a separation of the averaged formation latitude in the groups. The averaged $\mathrm{CE}$ formation latitudes in NN and NP groups are at least $5^{\circ} \mathrm{N}$ than that of $\mathrm{PP}$ and $\mathrm{PN}$ groups. There is an indication that the $\mathrm{NN}$ group may be influenced by a strengthening shear, but the PP group also experiences a strengthening shear albeit at a smaller rate. For the PN group that contains one-half of the entire cases, the shear remains nearly constant. The relationship with SST is also not straightforward. The NN group again shows a possible relationship with a SST decline of $1.8^{\circ} \mathrm{C}$, but this is less than the standard deviation of $3.3^{\circ} \mathrm{C}$. Both PN and PP show a decrease of around $1^{\circ} \mathrm{C}$, about the magnitude half of one standard deviation. A definitive 


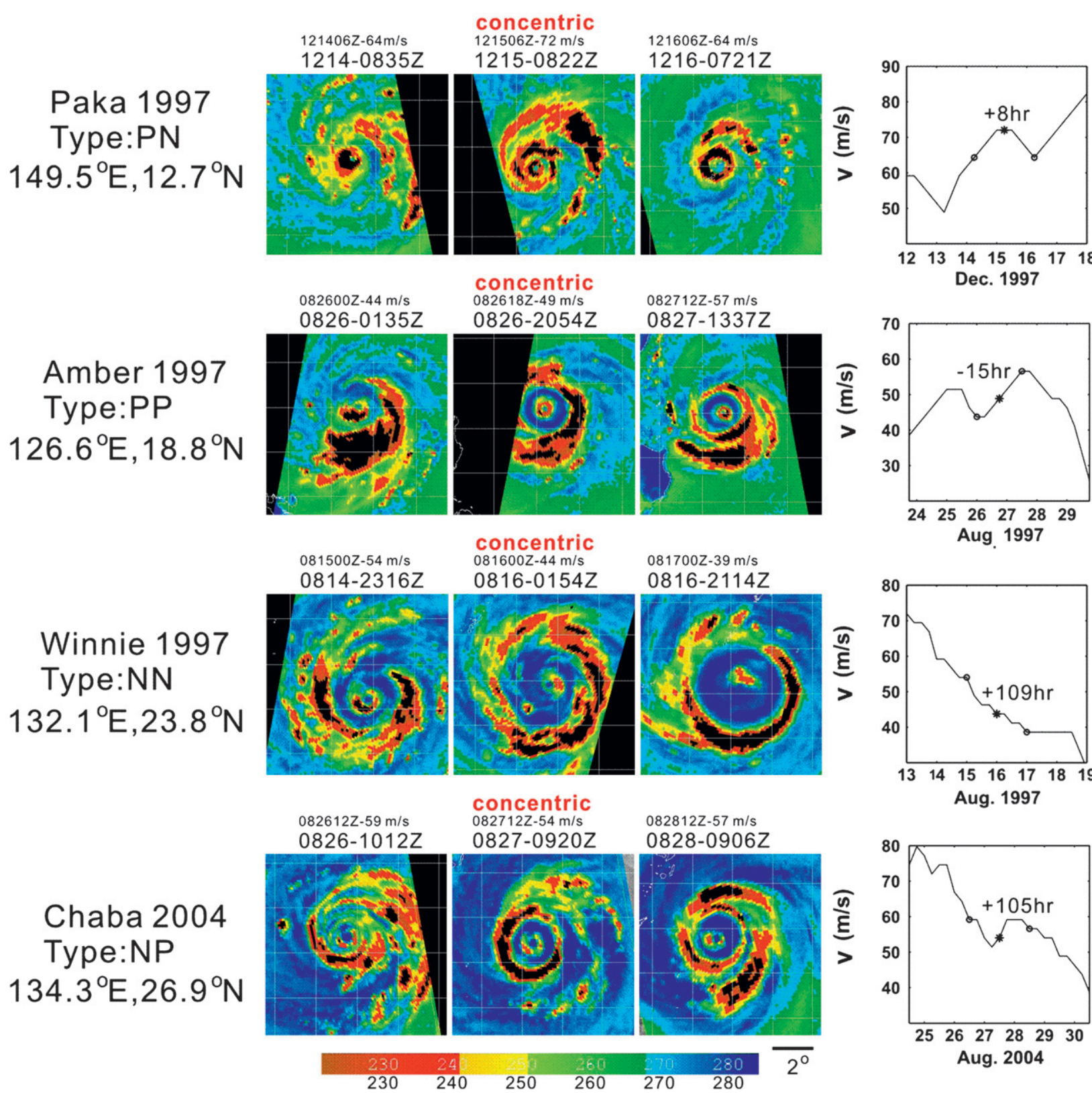

FIG. 9. Color-enhanced microwave images before, at, and after concentric eyewall formation for typical PN, PP, NN, and NP cases. The corresponding intensity time series is also shown. The length scale of $2^{\circ}$ in the satellite picture and the formation longitude and latitude are indicated. The numbers in the time series of intensity display the difference between the concentric eyewall formation time and the maximum intensity time.

relationship for the difference between groups and the environmental factors is therefore not obvious.

The intensity change between these groups may be mainly due to the CE dynamics. For example, the intensity in the PP case of Typhoon Amber (1997) appeared to increase from $24 \mathrm{~h}$ before to $24 \mathrm{~h}$ after the formation of the $\mathrm{CE}$ structure. The microwave imagery also indicates that the CE structure was maintained for at least $24 \mathrm{~h}$ after the formation, with no eyewall replacement cycle observed. Furthermore, the NN and NP cases often show a larger moat size (45 km averaged over $11 \mathrm{NN}$ cases and $50 \mathrm{~km}$ averaged over $2 \mathrm{NP}$ cases) and their inner eyewalls still identifiable for a period of $24 \mathrm{~h}$.

We compare the intensity change in the $\mathrm{CE}$ cases (with eyewall replacement cycle) with that of nonconcentric cases (no eyewall replacement cycle) in Fig. 10. For the concentric time series the composites are relative to the time of $\mathrm{CE}$ formation $(0 \mathrm{~h})$ while for the nonconcentric 
TABLE 1. The averages and standard deviations of vertical wind shear (850-200 and 850-500 hPa), SST, and formation latitude for the intensity change groups.

\begin{tabular}{|c|c|c|c|c|c|c|c|c|c|c|c|}
\hline \multicolumn{2}{|c|}{ Environment factors } & \multicolumn{3}{|c|}{ Vertical shear $(\mathrm{kt})(850-200 \mathrm{hPa})$} & \multicolumn{3}{|c|}{ Vertical shear $(\mathrm{kt})(850-500 \mathrm{hPa})$} & \multicolumn{3}{|c|}{$\operatorname{SST}\left({ }^{\circ} \mathrm{C}\right)$} & \multirow[b]{2}{*}{ Formation la } \\
\hline Groups & Time & $-24 \mathrm{~h}$ & $0 \mathrm{~h}$ & $24 \mathrm{~h}$ & $-24 \mathrm{~h}$ & $0 \mathrm{~h}$ & $24 \mathrm{~h}$ & $-24 \mathrm{~h}$ & $0 \mathrm{~h}$ & $24 \mathrm{~h}$ & \\
\hline \multirow[t]{2}{*}{ PN (22 cases) } & Avg & 9.7 & 9.9 & 10.7 & 6.5 & 4.9 & 5.4 & 29.0 & 28.6 & 28.1 & $20.2^{\circ} \mathrm{N}$ \\
\hline & Std dev & 5.2 & 4.7 & 9.2 & 5.8 & 4.0 & 6.0 & 0.6 & 0.7 & 0.8 & $4.7^{\circ}$ \\
\hline \multirow[t]{2}{*}{ PP (10 cases) } & Avg & 11.7 & 11.2 & 14.1 & 4.4 & 3.4 & 5.4 & 29.0 & 28.8 & 28.3 & $18.4^{\circ} \mathrm{N}$ \\
\hline & Std dev & 3.7 & 6.0 & 9.7 & 4.7 & 1.8 & 3.4 & 0.5 & 0.7 & 1.1 & $2.5^{\circ}$ \\
\hline \multirow[t]{2}{*}{ NN (8 cases) } & Avg & 9.2 & 11.7 & 17.1 & 3.2 & 4.6 & 7.0 & 28.7 & 28.3 & 26.9 & $25.7^{\circ} \mathrm{N}$ \\
\hline & Std dev & 6.1 & 6.9 & 12.5 & 3.7 & 3.8 & 5.5 & 0.6 & 0.7 & 2.7 & $4.3^{\circ}$ \\
\hline NP ( 2 cases $)$ & Avg & 16.1 & 9.0 & 14.3 & 5.2 & 3.8 & 5.6 & 28.4 & 28 & 27.6 & $26.4^{\circ} \mathrm{N}$ \\
\hline
\end{tabular}

cases they are relative to the peak intensity time $(0 \mathrm{~h})$. Figure 10 also gives the normalized intensity change. The normalized intensity change measures the relative intensity change of TCs in different categories. The nor- malization for the nonconcentric cases is with their life time peak intensity and the normalization for the concentric cases is with their intensity at the time of CE formation. Figures 10a,b are for stronger typhoon (categories (a)

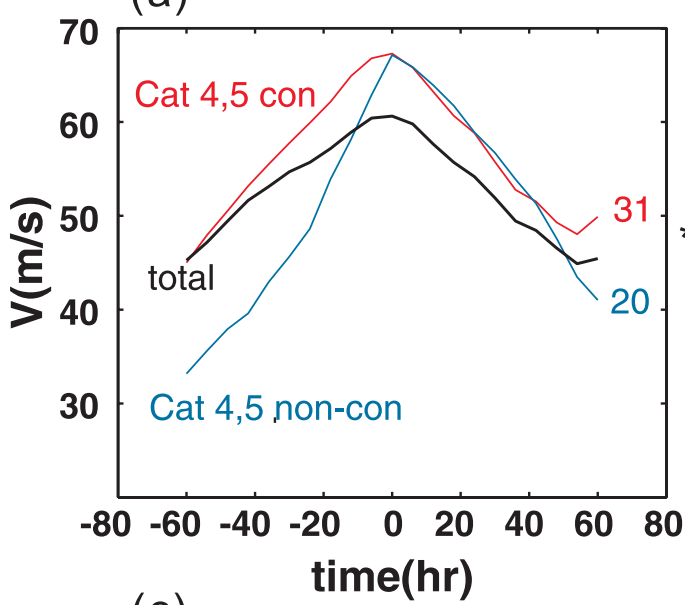

(c)

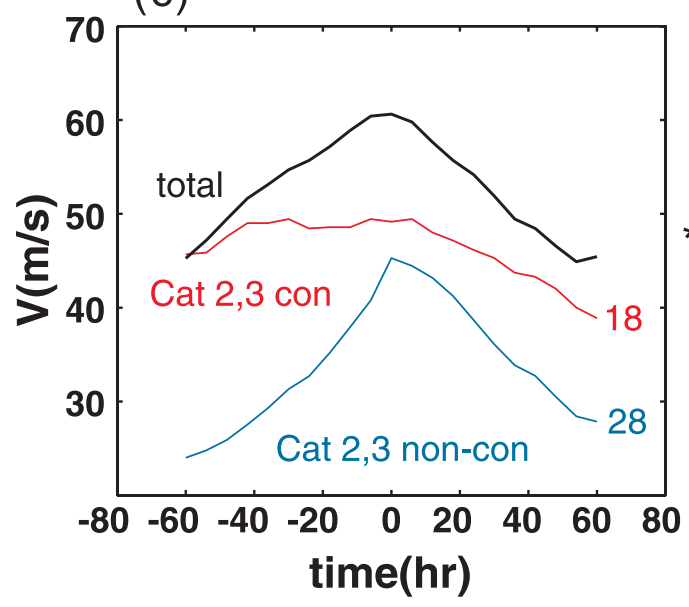

(b)

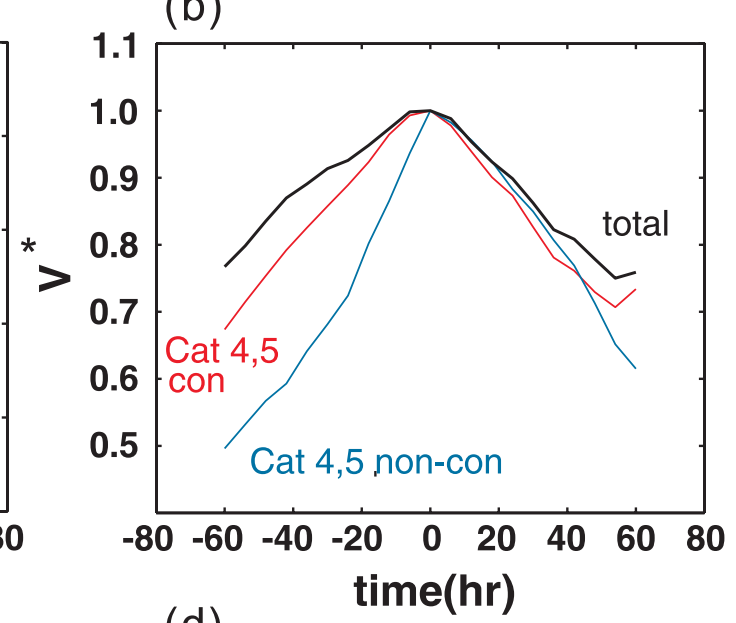

(d)

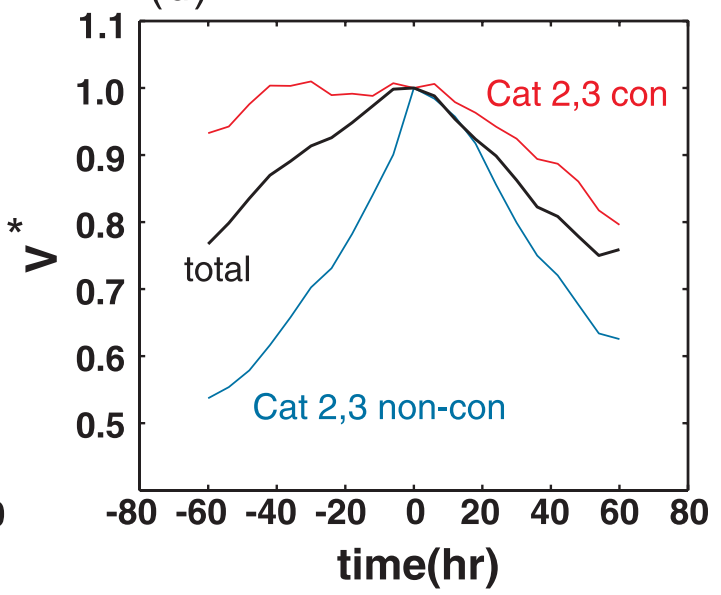

FIG. 10. Composite time series of intensity for the concentric and nonconcentric cases. Compositing was done relative to the time of concentric eyewall formation (maximum intensity) for the concentric typhoon cases (nonconcentric typhoon cases). (a),(b) The composite time series of intensity and normalized intensity for category 4 and 5 typhoons, respectively. (c),(d) As in (a),(b), but for category 2 and 3 typhoons. The total curves are the composites of concentric cases in categories $2-5$. 


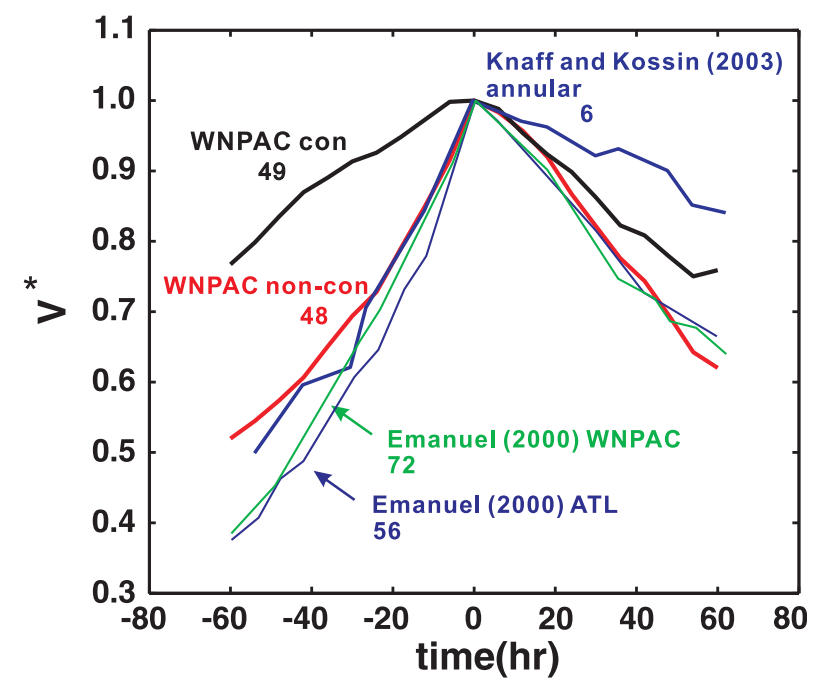

FIG. 11. Composite time series of the normalized intensity for the concentric and nonconcentric cases in the western North Pacific. Also plotted are the normalized intensity associated with average tropical cyclones in the Atlantic and western North Pacific that did not encounter cold water or make landfall as reported by Emanuel (2000) and annular hurricanes as reported by Knaff and Kossin (2003). Compositing was done relative to the time of concentric eyewall formation (maximum intensity) for the concentric (nonconcentric cases).

4 and 5) CE formation cases and the Figs. 10c,d are for weaker typhoons (categories 2 and 3 ), with the left panels showing the intensity changes and the right panels the normalized intensity changes. The "total" curve is the composites of concentric cases in categories 2-5.

Figure 10 shows that the composite intensity tendency for all $\mathrm{CE}$ typhoons prior to formation is distinctively different from the nonconcentric typhoons prior to reaching maximum intensity. The concentric composite reaches a higher normalized intensity prior to formation than the nonconcentric composite prior to peak intensity. However, the intensity tendencies for both strong concentric and nonconcentric typhoons are similar in the weakening phase. There is also some difference between the strong (categories 4 and 5) and weak (categories 2 and 3) CE typhoons, in that the former undergo a period of sustained intensification while the latter more or less just maintain their intensity with only modest intensification. The weakening tendencies in the weak cases of CE formation, however, are slower than in the strong cases. Compared to the non-CE cases, the formation of $\mathrm{CE}$ cases depends on the maintenance of a relatively high intensity for a longer duration prior to formation, rather than a rapid intensification process that can reach a higher intensity.

Figure 11 shows composite time series of the normalized intensity for the concentric and nonconcentric cases in the western North Pacific in addition to the normalized intensity associated with the average tropical cyclones in the Atlantic and western North Pacific that did not encounter cold water or make landfall as reported by Emanuel (2000), and annular hurricanes reported by Knaff and Kossin (2003). In our results, the cases near landfall were excluded. The intensity tendencies of our nonconcentric cases are in general agreement with Emanuel (2000). Compared to the non-CE typhoon composite, the concentric composite shows a relatively high intensity for a longer duration, rather than a rapid intensification process that can reach a higher intensity. Kuo et al. $(2004,2008)$ hypothesized that axisymmetrization of positive vorticity perturbations around a strong and tight core of vorticity may lead to the formation of $\mathrm{CE}$ structure. The long duration of high intensity before secondary eyewall formation may provide a favorable environment for the axisymmetrization dynamics to operate.

\section{Summary}

Using the best-track data and passive microwave satellite imagery we studied western North Pacific typhoons with CE structure during 1997-2006. Our results are as follows:

1) Most of the CE cases occur between June and October. There is a northern seasonal migration of $\mathrm{CE}$ formation locations. The CE structures formed at weaker intensities are often located at higher latitudes.

2) Approximately one-quarter of the cases examined possessed CE structure; including $57 \%$ of category 4 and $72 \%$ of category 5 typhoons that possessed CE at some point during their lifetime. Approximately onethird of CE cases are formed at the time of maximum intensity and a quarter of the CE cases are formed within $30 \mathrm{~h}$ after the time of maximum intensity.

3) There is no significant relationship between core size, intensity, core vorticity, and moat width for typhoons with CE typhoons.

4) A theoretical filamentation moat width is calculated based on observed data and is compared with the satellite-estimated moat width. The filamentation moat width explains $40 \%$ of the variance of the satelliteobserved moat width in the group of typhoons with $\mathrm{CE}$ formation intensity greater than $130 \mathrm{kt}$. The results appear to suggest that the rapid filamentation dynamics may contribute to the organization of the moat in very strong typhoons.

5) The intensity of the CE typhoons tends to peak at the time of secondary eyewall formation but the standard model of intensification followed by weakening is valid for only half of the cases. Approximately $74 \%$ 
of the cases intensify $24 \mathrm{~h}$ before secondary eyewall formation and approximately $72 \%$ of the cases weaken $24 \mathrm{~h}$ after formation.

6) Depending on the intensity change $24 \mathrm{~h}$ before the $\mathrm{CE}$ formation, there appears to be a separation of the averaged formation latitude in the PN, PP, NN, and NP groups. The averaged CE formation latitudes in $\mathrm{NN}$ and NP groups (e.g., negative intensity change before $\mathrm{CE}$ formation) are at higher latitudes $\left(5^{\circ} \mathrm{N}\right)$ than that of PP and PN groups.

7) A major difference between the concentric cases and nonconcentric cases is the intensity and intensity change during the preformation or intensification stage. The concentric cases are marked by a relatively high intensity lasting for a longer duration prior to formation, rather than a rapid intensification process that can reach a higher intensity as is for most nonconcentric cases. The long duration of high intensity may provide a favorable environment for the axisymmetrization dynamics to operate in CE formation.

8) There is no significant difference in the decaying rate of intensity between the averaged concentric cases after formation and nonconcentric cases reaching peak intensity.

Acknowledgments. We thank Jeffrey Hawkins, B. McNoldy, Yu-Ming Tsai, and reviewers for their helpful comments and suggestions. We thank Drs. M. DeMaria and J. A. Knaff for providing the Statistical Hurricane Intensity Prediction Scheme (SHIPS) data. The satellite microwave images were made available by the Naval Research Laboratory Marine Meteorology Division in Monterey, California. This research was supported by Taiwan's National Research Council through Grants NSC96-2111-M-002-002, NSC97-2628-M-002023, 97R0066-69, and MOTC-CWB-96-2M-01 to National Taiwan University, and by the U.S. Office of Naval Research to Naval Postgraduate School, under Award N0001408WR20125.

\section{REFERENCES}

Black, M. L., and H. E. Willoughby, 1992: The concentric eyewall cycle of Hurricane Gilbert. Mon. Wea. Rev., 120, 947-957.

DeMaria, M., M. Mainelli, L. K. Shay, J. A. Knaff, and J. Kaplan, 2005: Further improvement to the Statistical Hurricane Intensity Prediction Scheme (SHIPS). Wea. Forecasting, 20, 531-543.

Dodge, P., R. W. Burpee, and F. D. Marks Jr., 1999: The kinematic structure of a hurricane with sea level pressure less than $900 \mathrm{mb}$. Mon. Wea. Rev., 127, 987-1004.

Emanuel, K., 2000: A statistical analysis of tropical cyclone intensity. Mon. Wea. Rev., 128, 1139-1152.
Hawkins, J. D., and M. Helveston, 2004: Tropical cyclone multiple eyewall characteristics. Preprints, 26th Conf. on Hurricane and Tropical Meteorology, Miami, FL, Amer. Meteor. Soc., 276-277.

_ T. F. Lee, F. J. Turk, C. Sampson, J. Kent, and K. Richardson, 2001: Real-time Internet distribution of satellite products for tropical cyclone reconnaissance. Bull. Amer. Meteor. Soc., 82, 567-578.

—, M. Helveston, T. F. Lee, F. J. Turk, K. Richardson, C. Sampson, J. Kent, and R. Wade, 2006: Tropical cyclone multiple eyewall characteristics. Preprints, 27th Conf. on Hurricane and Tropical Meteorology, Monterey, CA, Amer. Meteor. Soc., 6B.1. [Available online at http://ams.confex. com/ams/27Hurricanes/techprogram/paper_108864.htm.]

Houze, R. A., S.-S. Chen, B. F. Smull, W.-C. Lee, and M. M. Bell, 2007: Hurricane intensity and eyewall replacement. Science, 315, 1235-1239.

Knaff, J. A., and J. P. Kossin, 2003: Annular hurricanes. Wea. Forecasting, 18, 204-223.

Kossin, J. P., and M. Sitkowski, 2009: An objective model for identifying secondary eyewall formation in hurricanes. Mon. Wea. Rev., 137, 876-892.

Kummerow, C., W. Barnes, T. Kozu, J. Shiue, and J. Simpson, 1998: The Tropical Rainfall Measuring Mission (TRMM) sensor package. J. Atmos. Oceanic Technol., 15, 809-817.

Kuo, H.-C., L.-Y. Lin, C.-P. Chang, and R. T. Williams, 2004: The formation of concentric vorticity structures in typhoons. J. Atmos. Sci., 61, 2722-2734.

— W. H. Schubert, C.-L. Tsai, and Y.-F. Kuo, 2008: Vortex interactions and barotropic aspects of concentric eyewall formation. Mon. Wea. Rev., 136, 5183-5198.

Maclay, K. S., M. DeMaria, and T. H. Vonder Haar, 2008: Tropical cyclone inner-core kinetic energy evolution. Mon. Wea. Rev., 136, 4882-4898.

Mallen, K. J., M. T. Montgomery, and B. Wang, 2005: Reexamining the near-core radial structure of the tropical cyclone primary circulation: Implications for vortex resiliency. J. Atmos. Sci., 62, 408-425.

Montgomery, M. T., and R. J. Kallenbach, 1997: A theory for vortex Rossby-waves and its application to spiral bands and intensity changes in hurricane. Quart. J. Roy. Meteor. Soc., 123, 435-465.

Nong, S., and K. A. Emanuel, 2003: A numerical study of the genesis of concentric eyewalls in hurricane. Quart. J. Roy. Meteor. Soc., 129, 3323-3338.

Rozoff, C. M., W. H. Schubert, B. D. McNoldy, and J. P. Kossin, 2006: Rapid filamentation zones in intense tropical cyclones. J. Atmos. Sci., 63, 325-340.

,-- , and J. Kossin, 2008: Some dynamical aspects of tropical cyclone concentric eyewalls. Quart. J. Roy. Meteor. Soc., 134, 583-593.

Samsury, C. E., and E. J. Zipser, 1995: Secondary wind maximum in hurricanes: Airflow and relationship to rainbands. Mon. Wea. Rev., 123, 3502-3517.

Shea, D. J., and W. M. Gray, 1973: The hurricane's inner core region. I. Symmetric and asymmetric structure. J. Atmos. Sci., 30, 1544-1564.

Wang, Y., 2008: Rapid filamentation zone in a numerically simulated tropical cyclone. J. Atmos. Sci., 65, 1158-1181.

Willoughby, H. E., 1990: Temporal changes of the primary circulation of tropical cyclones. J. Atmos. Sci., 47, 242-264. 